\section{Providing mothers with mobile phone message reminders increases childhood immunization and vitamin A supplementation coverage in Côte d'Ivoire: A randomized controlled trial}

\author{
Romance Dissieka, ${ }^{1}$ Marissa Soohoo, ${ }^{2}$ \\ Amynah Janmohamed, ${ }^{3}$ David Doledec ${ }^{4}$ \\ ${ }^{1}$ Helen Keller International, Abidjan, \\ Côte d'Ivoire; ${ }^{2}$ Ontario Public Service, \\ Toronto, ON, Canada; ${ }^{3}$ Independent \\ Consultant, Mississauga, ON, Canada; \\ ${ }^{4}$ Helen Keller International, Isaac \\ Gathanju Road, Nairobi, Kenya
}

\begin{abstract}
We conducted a randomized controlled trial to assess the effect of providing mothers with mobile voice or text (SMS) reminder messages on health facility attendance at five infant immunization and vitamin A supplementation (VAS) visits. The study was conducted at 29 health facilities in Korhogo district. Mothers were randomized to receive a voice or text reminder message two days prior to each scheduled visit and two additional reminders for missed doses ( $\mathrm{n}=798$; intervention group), or no phone reminder messages $(n=798$; control group). Infants in the intervention group were 2.85 (95\% CI: 1.85-4.37), 2.80 (95\% CI: $1.88-4.17), 2.68$ (95\% CI: 1.84- 3.91), and 4.52 (95\% CI: 2.84-7.20) times more likely to receive pentavalent 1-3 and MMR/yellow fever doses, respectively, and 5.67 (95\% CI: 3.48-9.23) times more likely to receive VAS, as compared to the control group. In the reminder group, $58.3 \%$ of infants completed all five visits, compared to $35.7 \%$ in the control group $(\mathrm{P}<0.001)$. Providing mothers mobile phone message reminders is a potentially effective strategy for improving immunization and VAS coverage in Côte d'Ivoire.
\end{abstract}

\section{Introduction}

Despite substantial improvements in recent decades, global childhood immunization coverage has stalled at $\sim 85 \%$, with coverage remaining far from optimal in several countries, placing numerous children at risk for morbidity and mortality associated with vaccine-preventable diseases. ${ }^{1}$ It is estimated close to 20 million infants do not receive routine EPI services. ${ }^{1}$ Sub-Saharan
Africa (SSA) has consistently had the lowest immunization coverage globally, with lack of knowledge about immunization schedules, weak health systems, low trust in health authorities, concerns about vaccine safety, and limited access to health facilities associated with low coverage in the region. ${ }^{2}$ In West Africa, high dropout rates between vaccine doses require strengthening of immunization delivery systems in the region. ${ }^{3}$

In addition to routine infant immunizations, vitamin A supplementation (VAS) for children 6-59 months has been a cornerstone of global child survival programs for decades, with more than 80 countries now having national VAS programs in place., ${ }^{4,5}$ In SSA, VAS has mainly been provided during National Immunization Days (NID) and polio Supplemental Immunization Activities. ${ }^{6,7}$ As these involve door-to-door provision of VAS, coverage has generally been high $(>$ $80 \%$ ), in contrast to the lower coverage achieved through health facility-based VAS delivery. ${ }^{8}$ More recently, semiannual Child Health Days (CHD) have become a key platform for VAS delivery in SSA. ${ }^{9}$ However, funding constraints and successful polio eradication efforts are shifting policies and programs away from NID and CHD campaigns towards integrating VAS into routine facility-based immunization services, thereby posing major challenges for sustaining high coverage levels. ${ }^{10-12}$

Since 2010, Helen Keller International (HKI) has been supporting the integration of VAS into routine facility immunization services in several SSA countries. This has involved introducing a 6-month VAS visit between the pentavalent 3 doses administered at 14 weeks and measles vaccine at 9 months of age. ${ }^{13}$ This makes VAS available year-round to age-eligible children, in contrast to the episodic nature of CHDs that occur every 4 to 6 months, which may miss infants turning 6 months between campaign rounds.

Mobile phone interventions, including short message service (SMS) appointment reminders, have shown to be effective for improving immunization coverage in low and middle-income countries. ${ }^{14-16}$ In 2012 , HKI began supporting the government of Côte d'Ivoire in the use of mobile phone messaging to increase immunization coverage, given that only $40 \%$ of children 12 months of age were estimated to be fully vaccinated in the country. ${ }^{17} \mathrm{~A}$ pilot study was conducted in Korhogo district that involved providing SMS reminders to mothers prior to each routine child immunization and newly-introduced VAS visit. The study revealed SMS reminder messages were not effective for increasing facil-
Correspondence: David Doledec, Helen Keller International, Isaac Gathanju Road, Nairobi, Kenya.

Tel: +254.718.397.967

E-mail: ddoledec@hki.org

Key words: infant, immunization, vitamin A, mHealth, Côte d'Ivoire.

Acknowledgements: the authors gratefully acknowledge the support and collaboration of the Cote d'Ivoire Ministry of Health and Public Hygiene, Expanded Program on Immunization, National Nutrition Program, and Korhogo Health District management and health providers who conducted study activities. We thank the mothers and caregivers who participated in the study.

Contributions: RD designed the study and coordinated the study activities; MS and AJ provided technical support for the analysis; DD provided technical oversight for all aspects of the study.

Conflict of interest: the authors declare no potential conflict of interest.

Funding: none.

Received for publication: 17 January 2019.

Revision received:16 April 2019.

Accepted for publication: 3 May 2019.

This work is licensed under a Creative Commons Attribution NonCommercial 4.0 License (CC BY-NC 4.0).

(C) Copyright R. Dissieka et al., 2019

Licensee PAGEPress, Italy

Journal of Public Health in Africa 2019; 10:1032 doi:10.4081/jphia.2019.1032

ity attendance due to low literacy and/or messages being missed. In July 2014, a second study was implemented in which mothers were given the option to receive either SMS or voice message reminders prior to scheduled visits. We examined the effect of providing these reminder messages to mothers on attendance at five infant immunization and VAS visits in Korhogo district.

\section{Materials and Methods}

\section{Study design and sampling method-} ology

The study was a randomized controlled trial conducted at 29 health facilities in Korhogo district in the north-central region of Côte d'Ivoire. Mothers (or caretakers) in the intervention group were provided SMS or voice message reminders, based on their 
preference, prior to each scheduled facility visit and two additional reminders in the event of non-attendance. Mothers in the control group were not provided any reminder messages. The five visits were: pentavalent 1 (DPT + Hep B + Hib) immunization at 6 weeks, pentavalent 2 at 10 weeks, pentavalent 3 at 14 weeks, VAS at 6 months, and MMR/yellow fever immunizations at 9 months of age delivered at publicsector facilities.

The sample size was based on detecting a $10 \%$ difference in coverage between the intervention and control groups, with $\beta=$ $0.80, \alpha=0.05$, and a $10 \%$ inflation factor to account for potential errors at registration (e.g., non-eligible participants). Block randomization was used to allocate motherchild pairs to the intervention or control group based on rural, semi-urban, or urban (Korhogo town) health facility location. Randomization was performed using an automated system and randomization schedules were concealed from health facility personnel. In each setting, purposive sampling was used to select health facilities based on sufficient population density and a well-functioning mobile phone network. Of the 29 facilities, 10 were located in rural areas, 12 in semi-urban areas, and 7 in Korhogo town.

\section{Participant recruitment}

During July 2014 to June 2015, motherchild pairs were recruited at all 29 health facilities at the time of the child's BCG immunization visit, which occurred within five weeks of the child's birth. If the mother was not present, the accompanying caretaker was invited to participate. To be eligible for the study, the mother/caretaker and child had to be residents of Korhogo district and the mother had to have primary access to a functioning mobile phone. Sociodemographic characteristics were collected for each mother-child pair by health facility staff at the time of enrollment. The study was approved by the Côte d'Ivoire National Research Ethics Committee (Comité national d'éthique et de la recherche). All mothers/caretakers provided written informed consent for their and the child's participation.

\section{Data collection and analysis}

At study enrollment, mothers were informed of subsequent dates for their infant's pentavalent 1-3 and MMR/yellow fever doses and VAS visit, which were also recorded on the child's health card that the mother took home. Dates for immunization sessions were arranged according to the EPI schedule ${ }^{13}$ and child's date of birth, excluding days when health facilities did not provide routine services (e.g., weekends, holi- days). Mothers in the intervention group were given the option to receive either an SMS or voice message reminder in their preferred language (French, Senoufo, or Dioula) prior to each scheduled visit. Messages were devised in collaboration with local EPI personnel and informed mothers to take their child for immunization or VAS in two days to protect the child's health. The content of SMS and voice messages was identical.

In the event the child was not brought to the facility on the scheduled date, another reminder was provided to the mother/caretaker three days after the missed appointment. If the child did not present a second time, a final reminder was sent two days before the next scheduled visit, in an attempt to catch the child up on the missed dose. Mothers in the control group were not provided any SMS or voice messages and were instructed to refer to the child's health card for appointment dates, as per the standard of care in Côte d'Ivoire. A mobile web service platform (Ivocarte ${ }^{\circledR}$, Abidjan) was used to register the mother's mobile phone number and child's visit schedule. SMS and voice messages were then automatically generated and transmitted to recipients at designated times. Attendance records for each visit were transmitted directly from facility staff to study personnel via the Ivocarte ${ }^{\circledR}$ platform. The study outcome measure was attendance at each visit and included children who were brought to the health facility on the scheduled appointment date or any other time during the study period. We conducted bivariate analyses and created multivariable logistic regression models to assess differences in visit attendance between the intervention and control group. Model covariates were selected based on a significant $(\mathrm{P}<0.05)$ bivariate result and/or factors associated with compliance. Covariates included maternal age, education, and employment; child age and gender; and health facility setting (rural, semi-urban, or urban). Results are presented as adjusted odds ratios (aOR) and $95 \%$ confidence intervals (CI) and all reported $\mathrm{P}$ values are 2-sided with a 0.05 significance level. Analyses were conducted using SPSS version 19.0 (Armonk, NY: IBM Corp) software.

\section{Results}

A total of 1596 mother-child pairs were enrolled in the study: 798 in the intervention group and 798 in the control group. Participant characteristics were similar between the two groups at recruitment (Table 1). Approximately half $(46.2 \% ; n=736)$ of all participants were enrolled at health facilities in Korhogo town, $35.0 \%(n=560)$ at facilities in semi-urban areas, and $18.8 \%$ $(n=300)$ at rural health facilities. The proportion of male and female children was similar in both study groups. The majority $(57.8 \% ; n=922)$ of mothers/caretakers were between 20-29 years of age, $68.0 \%$ $(\mathrm{n}=1090)$ had no formal education, and $69.7 \%(n=1112)$ reported having no formal employment. The percentage of mothers with no formal education was $92.3 \%$ $(n=679)$ in rural areas, $77.1 \%(n=431)$ in Korhogo town, and $51.8 \%(\mathrm{n}=155)$ in semiurban localities. In rural areas, $91.0 \%$ $(n=669)$ of mothers reported not working outside the home, as compared to $82.0 \%$ $(n=459)$ and $51.6 \%(n=154)$ in Korhogo town and semi-urban areas, respectively.

Among mothers in the intervention group, $84.6 \%(n=675)$ chose to receive a voice message reminder and $15.4 \%(n=123)$ opted to receive an SMS (text) message reminder prior to each child visit. Voice messages were preferred by $97.7 \%(n=293)$ of mothers in rural areas and $75.0 \%(n=552)$ in Korhogo town. Attendance at each visit was significantly higher in the reminder group, as compared to the control group (Table 2), with a mean difference of $17.4 \%$ and the largest differences occurring at the 6-month VAS (adjusted OR $=5.67 ; 95 \% \mathrm{CI}$ : 3.48-9.23) and 9-month MMR/yellow fever (adjusted $\mathrm{OR}=4.52 ; 95 \%$ CI: 2.84-7.20) sessions. Attendance was highest for the pentavalent 1 dose in both the intervention $(86.6 \% ; n=691)$ and control $(76.1 \% ; n=607)$ groups. While attendance decreased in both groups between the pentavalent 1 dose at 6 weeks and MMR/yellow fever immunization at 9 months, a larger overall decline was observed in the control group, with attendance reaching a low of $37.8 \%$ at the 9-month visit. More than half (58.3\%; $\mathrm{n}=465$ ) of infants in the reminder group completed all five visits and were, therefore, fully immunized and supplemented with a dose of vitamin A, compared to $35.7 \%$ $(\mathrm{n}=285)$ in the control group $(\mathrm{P}<0.001)$. In both groups, the percentage of children attending all visits was highest at facilities in semi-urban areas (intervention: 68.6\%; control: $52.5 \%$ ), followed by rural areas (intervention: $60.7 \%$; control: $36.0 \%$ ), and facilities in Korhogo town (intervention: $43.8 \%$; control: $16.6 \%$ ). Infants in semi-urban areas were more likely to receive $\operatorname{VAS}(\mathrm{OR}=1.60$; 95\% CI: 1.17-2.21) and MMR/yellow fever doses $(\mathrm{OR}=1.55 ; 95 \% \mathrm{CI}: 1.14-2.12)$, as compared to children in rural areas (Table 3). Infants in Korhogo town were less likely to receive pentavalent $1(\mathrm{OR}=0.22 ; 95 \%$ CI: 0.14-0.36), pentavalent $2(\mathrm{OR}=0.28 ; 95 \%$ CI: $0.19-0.41)$, pentavalent $3(\mathrm{OR}=0.37 ; 95 \%$ 
CI: $0.26-0.52$ ), VAS (OR $=0.39$; $95 \%$ CI: 0.28 $0.54)$, and $\mathrm{MMR} /$ yellow fever $(\mathrm{OR}=0.56 ; 95 \%$ CI: 0.40-0.79), as compared to children in rural areas. There was no significant difference in attendance at any visit based on the type (SMS vs. voice) of message provided.

\section{Discussion and Conclusions}

In this study, providing mothers with mobile phone reminder messages increased the proportion of children receiving routine immunizations and vitamin A supplementation. Voice or SMS reminder messages prior to scheduled visits resulted in $20 \%$ more fully immunized and vitamin A-supplemented children, compared to the control group. As expected, attendance was higher in semi-urban, compared to rural, areas in both groups. However, the fact that $60 \%$ of infants in the intervention group attended all five visits in rural areas suggests providing telephone reminders is a feasible and acceptable approach for increasing immunization and VAS compliance in harder-toreach communities. The fact that infants in Korhogo town were least likely to be brought to immunization and VAS sessions may in part be due to mothers' reliance on nannies and other family members for child care, including preventive health services, in urban areas.

The majority of mothers preferred to receive voice reminders rather than SMS messages. This was likely due to a preference for oral communication channels, a lack of familiarity with text messaging (particularly in rural areas), and challenges related to reading and understanding written messages, given that $\sim 70 \%$ of mothers had no formal education. Low levels of educa- tion and literacy have shown to be barriers to caregiver access to text message-based interventions in SSA. ${ }^{18}$ Though we did not assess the literacy level of our study population, the 2011-2012 DHS indicated 46\% and $78 \%$ of women in urban and rural areas, respectively, are illiterate in Côte d'Ivoire. ${ }^{17}$ Therefore, visual images reminding moth- ers to attend immunization sessions may be a viable alternative to providing text messages in this context. The fact that attendance was similar between male and female children in urban and rural areas is encouraging and suggests gendered decision-making for child immunization and VAS was not apparent in these settings.

Table 1. Participant characteristics at enrollment.

\begin{tabular}{lrrr} 
& Intervention No. (\%) & Control No. (\%) & Total No. (\%) \\
Area & & & \\
$\quad$ Rural & $150(9.4)$ & $150(9.4)$ & $300(18.8)$ \\
Semi-urban & $280(17.5)$ & $280(17.5)$ & $560(35.0)$ \\
Korhogo town & $368(23.1)$ & $368(23.1)$ & $736(46.2)$ \\
Mother's education & & & \\
No education & $541(67.8)$ & $549(68.8)$ & $1090(68.0)$ \\
Primary & $123(15.4)$ & $107(13.4)$ & $230(14.0)$ \\
Secondary & $117(14.7)$ & $118(14.8)$ & $235(15.0)$ \\
Higher & $17(2.1)$ & $24(3.0)$ & $41(3.0)$ \\
\hline Mother's language & & & \\
Senoufo & $460(58.6)$ & $471(60.0)$ & $931(58.3)$ \\
Dioula & $230(29.3)$ & $209(26.6)$ & $439(27.5)$ \\
French & $55(7.0)$ & $67(8.5)$ & $122(7.6)$ \\
Other & $53(6.8)$ & $51(6.5)$ & $104(6.5)$ \\
Mother's occupation & & & $1112(69.7)$ \\
Unpaid/homemaker & $551(69.0)$ & $561(70.3)$ & $325(20.4)$ \\
Business & $165(20.7)$ & $160(20.1)$ & $80(5.0)$ \\
Employed & $39(4.9)$ & $41(5.1)$ & $79(4.9)$ \\
Other & $43(5.4)$ & $36(4.5)$ & \\
\hline Mother's age (years) & & & $178(11.2)$ \\
$\leq 19$ & $99(12.4)$ & $79(9.9)$ & $436(27.3)$ \\
$20-24$ & $210(26.3)$ & $226(28.3)$ & $486(30.5)$ \\
$25-29$ & $234(29.3)$ & $252(31.6)$ & $345(21.6)$ \\
$30-34$ & $172(21.6)$ & $173(21.7)$ & $128(8.0)$ \\
$35-39$ & $68(8.5)$ & $60(7.5)$ & $23(1.0)$ \\
$\geq 40$ & $15(1.9)$ & $8(1.0)$ & $792(49.6)$ \\
Child sex & $401(50.3)$ & $391(49.0)$ & \\
Female & $397(49.7)$ & $407(51.0)$ & \\
Male & & \\
\hline
\end{tabular}

$\mathrm{N}=1596$ mother-child pairs; $\mathrm{P}>0.05$ for all variables (chi-squared test)

Table 2. Immunization and vitamin A supplementation visit attendance.

\begin{tabular}{|c|c|c|c|c|}
\hline Facility visit & Intervention No. (\%) & Control No. (\%) & aOR $(95 \%$ CI) & P value \\
\hline Pentavalent $1^{\mathrm{a}}(6 \mathrm{wk})$ & $691(86.6)$ & $607(76.1)$ & $2.85(1.85-4.37)$ & $<0.001$ \\
\hline Pentavalent $2^{\mathrm{a}}$ (10 wk) & $646(81.0)$ & $537(67.3)$ & $2.80(1.88-4.17)$ & $<0.001$ \\
\hline Pentavalent $3^{\mathrm{a}}$ (14 wk) & $592(74.2)$ & $465(58.3)$ & $2.68(1.84-3.91)$ & $<0.001$ \\
\hline Vitamin A (6 mo) & $516(64.7)$ & $325(40.7)$ & $5.67(3.48-9.23)$ & $<0.001$ \\
\hline MMRb/yellow fever (9 mo) & $484(60.7)$ & $302(37.8)$ & $4.52(2.84-7.20)$ & $<0.001$ \\
\hline
\end{tabular}

CI: confidence interval; aOR: adjusted odds ratio; $\mathrm{N}=1596$ mother-child pairs. Models adjusted for: mother's age, language, occupation, education; child age and gender; facility location (rural, semi-urban, town), type

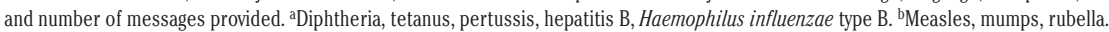

Table 3. Immunisation and vitamin A supplementation visit attendance according to health facility location.

\begin{tabular}{|c|c|c|c|c|c|}
\hline Health facility location & $\begin{array}{l}\text { Pentavalent } 1^{\mathrm{a}} \\
\text { OR }(95 \% \mathrm{CI})\end{array}$ & $\begin{array}{l}\text { Pentavalent } 2^{\mathrm{a}} \\
\text { OR }(95 \% \mathrm{CI})\end{array}$ & $\begin{array}{l}\text { Pentavalent } 3^{\mathrm{a}} \\
\text { OR }(95 \% \mathrm{CI})\end{array}$ & $\begin{array}{c}\text { Vitamin A } \\
\text { OR (95\% CI) }\end{array}$ & $\begin{array}{c}\text { MMR } \mathrm{b} / \text { yellow fever } \\
\text { OR }(95 \% \mathrm{CI})\end{array}$ \\
\hline Korhogo town & $0.22(0.14-0.36)$ & $0.28(0.19-0.41)$ & $0.37(0.26-0.52)$ & $0.39(0.28-0.54)$ & $0.56(0.40-0.79)$ \\
\hline Semi-urban & $1.00(0.60-1.64)$ & $1.10(0.74-1.66)$ & $1.41(0.99-2.00)$ & $1.60(1.17-2.21)$ & $1.55(1.14-2.12)$ \\
\hline Rural (ref) & Reference & Reference & Reference & Reference & Reference \\
\hline
\end{tabular}

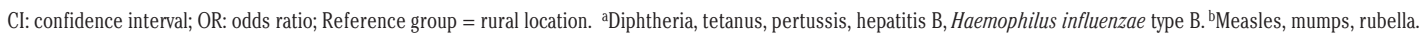


The positive impact of reminder messages in our study is likely due to various reasons. Mothers were reminded about appointments that were scheduled well in advance, which they might have otherwise forgotten. Also, receiving the automated messages may have increased trust and confidence in the health system, thereby making mothers more inclined to seek care for their children. Receiving multiple reminder messages may have instilled a greater sense of obligation on the part of mothers to bring their children for immunizations and VAS.

Our findings are consistent with evidence showing mobile reminders have led to improvements in immunization coverage and decreased dropout rates between doses in Sub-Saharan Africa. ${ }^{19}$ A trial in Senegal revealed SMS reminder messages to caretakers resulted in $>60 \%$ higher VAS coverage at a 6-month facility visit, with twofold higher coverage in rural compared to urban districts. ${ }^{20}$ In Nigeria, phone call reminders to mothers two days and one day before scheduled appointments were effective for increasing infant immunization completion, compared to a control group $(\mathrm{RR}=1.72$; 95\% CI: $1.50-1.98) .{ }^{21}$ Finally, in a study conducted in Zimbabwe, immunization coverage at 6 weeks was $97 \%$ for a group receiving SMS reminder messages, compared to $82 \%$ in a non-intervention group, and $95 \%$ vs. $75 \%$ at the 14 -week visit in the reminder and control group, respectively. ${ }^{22}$

In our study, though mobile reminders increased attendance at immunization and VAS sessions, overall compliance was suboptimal. The fact that $25 \%$ and $40 \%$ of infants in the reminder and control group, respectively, did not complete all five visits is concerning as it means a large percentage of children were not fully immunized and/or protected against the consequences of vitamin A deficiency during the first year of life. This is consistent with immunization trends in Côte d'Ivoire, with the most recent DHS indicating only $35.7 \%$ of children who received BCG vaccine were fully immunized at 12 months of age. ${ }^{17}$

Despite the provision of reminders, reasons for non-attendance in both urban and rural areas likely included the high cost of transport to the health facility and mothers not having the time to bring the child and or not having listened to or read the messages. Additionally, logistical factors such as change of phone number/SIM card, which commonly occur in such settings, could have affected attendance. Though we recruited women with primary access to a mobile phone, the practice of phone sharing within households was not investigated and could have influenced whether messages reached intended recipients.

Current data indicate global VAS coverage is at a 6-year low, with particularly sharp declines observed in West and Central Africa. ${ }^{23}$ In 2016, only 10 SSA countries reported high $(\geq 80 \%)$ recommended twodose VAS coverage. ${ }^{23,24}$ Furthermore, a recent study involving 28 SSA countries revealed only $66 \%$ of children $12-23$ months received at least one VAS dose. ${ }^{25}$ In our study, the $65 \%$ attendance for VAS at 6 months in the intervention group indicates potential for successfully integrating VAS into facility-based child health services.

A strength of our study was the randomized design with comparable groups at baseline. Health providers were blinded to the randomization scheme, which reduced the likelihood of bias in service delivery and data recording. Also, immunization and VAS data were uploaded to the web server in real-time, which enabled more efficient monitoring of each child's progress. This likely resulted in more complete and accurate data, as compared to self-reported data and child health cards. However, the recruitment of mother-child pairs at the time of the child's BCG vaccination may have resulted in a sample of mothers who were more proactive in health-seeking practices for their children and, consequently, may have introduced some bias into our sample. In addition, as 19 out of the 29 health facilities in this study also participated in the previous pilot intervention, health providers and communities in these areas may have been more sensitized to the importance of immunization and VAS, as compared to the other 10 facilities. As health facilities were purposively selected in areas with a well-functioning mobile phone network, the findings may not be generalizable to areas with poorer network coverage and where access to mobile phones may also be limited.

A reduction in child morbidity and mortality from vaccine-preventable diseases and vitamin A deficiency requires strengthening facility-based immunization and VAS delivery systems. A major barrier to achieving high coverage is the lack of communication between service providers and caregivers regarding the timing of essential facility visits. Findings from our study suggest the use of simple mobile messaging is feasible and effective for improving immunisation and VAS coverage in Côte d'Ivoire. With rapidly-increasing mobile phone penetration in the country, mobile-based interventions should be considered to strengthen child survival programs in Côte d'Ivoire.

\section{References}

1. WHO. WHO immunization coverage fact sheet; updated April 2018. Available from: http://origin.who.int/ mediacentre/factsheets/fs378/en/.

2. Esposito S, Principi N, Cornaglia G, ESCMID Vaccine Study Group (EVASG). Barriers to the vaccination of children and adolescents and possible solutions. Clin Microbiol Infect 2014;20:25-31.

3. Kazungu, JS, Adetifa I. Crude childhood vaccination coverage in West Africa: Trends and predictors of completeness. Wellcome Open Res 2017;2.

4. Vitamin A Supplementation: A Decade of Progress. New York: United Nations Children's Fund; 2007. Available from: https:/www.unicef.org/publications/ files/Vitamin_A_Supplementation.pdf.

5. Stevens GA, Bennett JE, Hennocq Q, et al. Trends and mortality effects of vitamin A deficiency in children in 138 low-income and middle-income countries between 1991 and 2013: A pooled analysis of population-based surveys. Lancet Glob Health 2015;3:e528-36.

6. Wirth JP, Petry N, Tanumihardjo SA, et al. Vitamin A supplementation programs and country-level evidence of vitamin A deficiency. Nutrients 2017;9:e190.

7. Integration of vitamin A supplementation with immunization: policy and programme implications. New York: United Nations Children's Fund; 1998. Available from: http://apps.who. int/iris/bitstream/ handle/10665/64149/ WHO_EPI_GEN_98.07.pdf?sequence $=1$.

8. Goodman T, Dalmiya N, de Benoist B, Schultink W. Polio as a platform: using national immunization days to deliver vitamin A supplements. Bull World Health Organ 2000;78:305-14.

9. Doherty T, Chopra M, Tomlinson M, et al. Moving from vertical to integrated child health programmes: experiences from a multi-country assessment of the Child Health Days approach in Africa. Trop Med Int Health 2010;15:296-305.

10. Palmer AC, Diaz T, Noordam AC, Dalmiya N. Evolution of the child health day strategy for the integrated delivery of child health and nutrition services. Food Nutr Bull 2013;34:4129.

11. Chehab ET, Anya BM, Onyango AW, et al. Experience of integrating vitamin A supplementation into polio campaigns in the African Region. Vaccine 
2016;34:5199-202.

12. Hodges MH, Sesay FF, Kamara HI, et al. Integrating vitamin A supplementation at 6 months into the Expanded Program of Immunization in Sierra Leone. Matern Child Health J 2015;19:1985-92.

13. WHO. WHO vaccination recommendations for children. Recommended Routine Immunizations for Children August 2018. Available from: https://www.who.int/immunization/policy/Immunization_routine_table2.pdf?u $\mathrm{a}=1$.

14. Barnett I, Yosellina, Sulistyo S, et al. Mixed-method impact evaluation of a mobile phone application for nutrition monitoring in Indonesia. Brighton, UK: Institute of Development Studies; 2016. Available from: https://www.ids.ac.uk/ publication/mixed-method-impactevaluation-of-a-mobile-phone-application-for-nutrition-monitoring-inindonesia.

15. Amoakoh-Coleman M, Borgstein AB, Sondaal SF, et al. Effectiveness of mHealth interventions targeting health care workers to improve pregnancy outcomes in low- and middle-income countries: A systematic review. J Med Internet Res 2016;18:e226.

16. Oliver-Williams C, Brown E, Devereux
$\mathrm{S}$, et al. Using mobile phones to improve vaccination uptake in 21 lowand middle-income countries: Systematic review. JMIR mHealth and uHealth 2017;5:e148.

17. Côte d'Ivoire 2011-2012 Enquête Démographique et de Santé et à Indicateurs Multiples. Abidjan, Côte d'Ivoire and Calverton, Maryland: Institut National de la Statistique and MEASURE DHS, ICF International; 2013. Available from: https://dhsprogram.com/ pubs/pdf/ FR272/FR272.pdf

18. Kazi AM, Carmichael JL, Hapanna GW, et al. Assessing mobile phone access and perceptions for textingbased mHealth interventions among expectant mothers and child caregivers in remote regions of Northern Kenya: A survey-based descriptive study. JMIR Public Health Surveill 2017;3:e5.

19. Manakongtreecheep K. SMS-reminder for vaccination in Africa: research from published, unpublished and grey literature. Pan Afr Med J 2017;27:23.

20. Thiaw C, Cooper A, Tendeng C, et al. Routine delivery of vitamin A supplementation at six months in Senegal using SMS reminder messages. EJNFS 2015;5:804-5.

21. Brown VB, Oluwatosin OA, Akinyemi
JO, Adeyemo AA. Effects of community health nurse-led intervention on childhood routine immunization completion in primary health care centers in Ibadan, Nigeria. J Community Health 2016;41:265-73.

22. Bangure D, Chirundu D, Gombe N, et al. Effectiveness of short message services reminder on childhood immunization programme in Kadoma, Zimbabwe - a randomized controlled trial, 2013. BMC Public Health 2015;15:137.

23. Coverage at a crossroads: New directions for vitamin A supplementation programmes. New York: UNICEF; 2018. Available from: https://www.unicef.it/Allegati/Coverag e _at_Crossroads_Vitamin_A.pdf.

24. WHO Guideline: Vitamin $\bar{A}$ supplementation in infants and children 6-59 months of age. Geneva: World Health Organization; 2011. Available from: http://apps.who.int/iris/bitstream/handle/10665/44664/9789241501767_eng. pdf;jsessionid=E6D48C94208400A479 082A98638BD121 ? sequence $=1$.

25. Anand A, Luman ET, O'Connor PM. Building on success- potential to improve coverage of multiple health interventions through integrated delivery with routine childhood vaccination. J Infect Dis 2012;205:S28-39. 